\title{
Stricture of Urethra: Patterns and Outcomes of Management from a Single Centre in Pakistan Over 7 Years
}

Manzoor Hussain, Muhammad Shamim Khan, Murli Lal, Altaf Hashmi, Syed Ali Anwer Naqvi and Syed Adibul Hasan Rizvi Department of Urology, Sindh Institute of Urology and Transplantation, Karachi, Pakistan

\begin{abstract}
Objective: To determine the outcomes of urethroplasty and its complications from a large cohort of patients managed in a single centre.

Study Design: Descriptive study.

Place and Duration of Study: Department of Urology, Sindh Institute of Urology and Transplantation (SIUT), Karachi, from January 2010 to December 2016.

Methodology: A total of 546 patients with stricture urethra at different locations underwent urethroplasty from January 2010 to December 2016 were included. All patients had an ascending urethrogram followed by retrograde \pm antegrade urethroscopy to assess the location and length of the stricture. Technique of urethroplasty was chosen according to the site, length and etiology. Following appropriate procedure, patients were followed up in the dedicated urethral stricture clinic. Procedure was considered successful if either no further therapeutic intervention was required and the maximum flow rate (Qmax) was $>20 \mathrm{ml} / \mathrm{sec}$ with a voided volume of at least $200 \mathrm{mls}$. The procedure was regarded as unsuccessful, if further treatment was required or Qmax was $<10 \mathrm{ml} / \mathrm{sec}$.

Results: A total of 546 patients with mean age of $32.3 \pm 13.1$ years (range: $12-74)$ involving anterior $(n=323,59.2 \%)$ or posterior $(n=223,40.8 \%)$ urethra were treated. Mean follow-up was 43.6 months (range: 3-84). The success rates of bulbar urethral strictures after excision and primary anastomosis (EPA) was $93.3 \%$, non-transecting urethroplasty $84.6 \%$ and oral mucosal graft (OMG), $81.8 \%$. In penile urethral strictures, OMG, Orandi procedure and Johanson's techniques yielded success rates of $88.4 \%, 66.6 \%$ and $57.1 \%$, respectively. In posterior urethral strictures, after excision and bulboprostatic anastomosis, good results were seen in $88.3 \%$. In pan-urethral strictures, abdominal skin graft repair, combined tissue transfer and OMG urethroplasty yielded success rates of $74 \%, 78.5 \%$ and $75 \%$, respectively. The complications/ adverse events were encountered in $251 / 546(45.9 \%)$ patients in this series.

Conclusion: Anastomotic urethroplasty yielded best outcomes followed by OMG urethroplasty. In the long-term follow-up, erectile dysfunction (ED), infertility and recurrence of stricture are the main complications which need individualised management.
\end{abstract}

Key Words: Urethroplasty, Outcomes, Complications.

How to cite this article: Hussain M, Khan MS, Lal M, Hashmi A, Naqvi SAA, Rizvi SAH. Stricture of urethra: Patterns and outcomes of management from a single centre in Pakistan over 7 years. J Coll Physicians Surg Pak 2020; 30(1):79-84.

\section{INTRODUCTION}

Urethral stricture management is a challenging discipline of reconstructive surgery due to variations in etiology, location, extent of fibrosis and prior multiple surgical interventions. ${ }^{1}$ The treatment options range from dilatation, direct visual internal urethrotomy (DVIU), laser incision, stents and urethroplasty. ${ }^{2-4}$ Urethroplasty is considered the gold standard as it yields best outcomes; restoration of normal voiding with lowest risk of recurrence compared to less invasive procedures. ${ }^{5}$

Correspondence to: Prof. Manzoor Hussain, Department of

Urology, Sindh Institute of Urology and Transplantation,

Civil Hospital, Karachi-74200, Pakistan

E-mail:hmanzoor2015@gmail.com

Received: March 04, 2019; Revised: June 27, 2019;

Accepted: June 27, 2019
There are three principal aims in urethroplasty; restoring normal voiding while preserving the erectile function and fertility. ${ }^{6}$ However, these objectives are generally not achievable in pan-urethral strictures irrespective of etiology and technique. Sexual dysfunction after urethroplasty has significant impact on patients' quality of life (QOL) and overall satisfaction. To reduce the risk of these complications, technique of non-transecting urethroplasty (NTU) has been proposed as an alternative in highly selected cases. ${ }^{7}$

Stricture management can be grouped into four anatomical domains; penile, bulbar, pan-urethral and posterior. The reconstructive procedures include excision and primary anastomosis (EPA), graft procedures and perinealurethrostomy. 8

This study aimed to report on the outcomes of the urethral stricture surgery and its complications in a single institution from Pakistan. 


\section{METHODOLOGY}

A descriptive study was performed from January 2010 to December 2016 at Sindh Institute of Urology and Transplantation (SIUT). During this period, 546 patients underwent urethroplasty. All patients gave informed consent to participate in the study. All patients with strictures in penile, bulbar, posterior urethra and panurethral involvement were included. Preoperative evaluation included history, physical examination, urinalysis, urine culture, renal function test, ascending urethrogram and voiding cysto-urethrogram. Data collected included, demographics, etiology, flow rate, urinalysis, culture results, renal profile, imaging and endoscopic assessment and technique of repair and complications.

Catheter was removed two weeks after an anterior and 3 weeks following posterior urethroplasty. Supra-pubic catheter (SPC) was clamped and uroflowmetry performed in all cases.

Different techniques were used according to the location of strictures as per standard guidelines.7,9,10

Success rate and complications were recorded at 3,6 and 12 months and at the end of the study period. The criteria used to assess the outcome was based on results of uroflow, measurement of post-void residual and flexible urethroscopy in cases with suboptimal flow rate results. If $\mathrm{Qmax}$ was $>20 \mathrm{ml} / \mathrm{sec}$, urethroplasty was successful. A flow rate in range of $10-15 \mathrm{ml} / \mathrm{sec}$ after the procedure was a fair result but if it was either $<10 \mathrm{ml} / \mathrm{sec}$ or patient required any additional intervention, procedure was a failure. Following successful trial of voiding, SPC was removed after a week. In patients undergoing two-stage procedures, the second stage being performed after three months. Patients with poor outcomes underwent either redo urethroplasty, laser urethrotomy or DVIU.

Follow-up in the urethral clinic ranged from 3 to 84 months. On each follow-up visit, patients were questioned about voiding, erectile function, infertility and overall satisfaction after urethroplasty in a questionnaire developed by consensus of local urologists.

SPSS version 20 was used to analyse the data. Descriptive statistics were used to summarise the continuous and categorical variables. Continuous variables like age and follow-up period were presented as mean \pm standard deviation (SD). Categorical variables such as location of stricture were expressed as frequencies with percentages. Chi-square test was applied to assess the differences in etiology and outcomes according to different locations and techniques of urethroplasty. A $p$-value of $<0.05$ was considered as significant.

\section{RESULTS}

A total of 3,357 procedures were performed for urethral stricture during the study period. Of these, 1,447 (43.1\%) underwent urethroscopy and dilatation, 1,133 (33.7\%) DVIU and $35(1.0 \%)$ laser urethrotomy. A total of 546 $(16.2 \%)$ male patients had urethroplasty. The mean age was $32.3 \pm 13.0$ years (range $=12-74$ years). Of these, $212(38.8 \%)$ had bulbar, $222(40.7 \%)$ pelvic fracture urethral injury (PFUI), 55 (10.1\%) penile and 57 (10.4\%) had pan-urethral location of stricture. The mean followup was 43.5 months (range: 3-84 months). The aetiology of different urethral strictures in $\mathbf{5 4 6}$ patients is shown in Table I. There were significant differences in the etiology of stricture depending on the location of the stricture in vast majority of cases, as shown by p-values in Table I. The outcomes are shown in Table II. There were no significant difference in success rates of outcome depending on various techniques in penile $(p=0.20)$, and bulbar $(p=0.06)$ urethral strictures. On the other hand, there were significant differences in the rates of success in pan-urethral strictures depending on the techniques of repair $(p=0.02)$, as shown in Table II.

The early adverse events and surgical complications in 546 patients are listed in Table III. Most of the complications were minor (grade-1) according to Clavian-Dindo classification. There were no statistically significant differences in complications in relation to the

Table I: Etiology of 546 urethral strictures according to location of stricture.

\begin{tabular}{|c|c|c|c|c|c|c|}
\hline & $\begin{array}{c}\text { Penile }(n=55) \\
n(\%)\end{array}$ & $\begin{array}{c}\text { Bulbur }(n=212) \\
n(\%)\end{array}$ & $\begin{array}{c}\text { Pan urethra }(n=57) \\
n(\%)\end{array}$ & $\begin{array}{c}\text { Posterior }(\mathrm{n}=222) \\
\mathrm{n}(\%)\end{array}$ & $\begin{array}{c}\text { Total } \\
\mathrm{n}\end{array}$ & $p$-value \\
\hline Idiopathic & $11(20.3)$ & $50(23.5)$ & $11(19.2)$ & $3(1.3)$ & 75 & $<0.001$ \\
\hline Straddle injury & $3(5.5)$ & $47(22.1)$ & $4(7)$ & $5(2.2)$ & 59 & $<0.001$ \\
\hline Post urethral catheter & $6(11.1)$ & $10(4.7)$ & $4(7)$ & $3(1.3)$ & 23 & $<0.008$ \\
\hline Post TURP & $1(1.8)$ & $7(3.3)$ & - & $3(1.3)$ & 11 & 0.32 \\
\hline Post hypospadias & $7(12.9)$ & $1(0.47)$ & $2(2.3)$ & - & 10 & $<0.001$ \\
\hline Road traffic accidents & $2(3.7)$ & $9(4.2)$ & $3(5.2)$ & $30(13.4)$ & 44 & 0.002 \\
\hline STI/urethitis & $4(7.4)$ & $3(1.4)$ & $8(14)$ & - & 15 & $<0.001$ \\
\hline BXO/lichen sclerosis & $8(14.8)$ & - & $4(7)$ & - & 12 & $<0.001$ \\
\hline Post instrument & $6(11.1)$ & - & $7(12.2)$ & - & 13 & $<0.001$ \\
\hline Penile trauma & $6(11.1)$ & - & $5(8.7)$ & - & 11 & $<0.001$ \\
\hline Pelvic fracture urethral injury & - & $3(1.4)$ & - & $208(93.2)$ & 211 & $<0.001$ \\
\hline Gun shot & $2(3.7)$ & $8(3.7)$ & - & $10(4.4)$ & 20 & 0.45 \\
\hline Water cane perineum & - & $3(1.4)$ & - & $2(0.8)$ & 5 & 0.65 \\
\hline
\end{tabular}


Table II: Outcomes of urethroplasty according to location and technique.

\begin{tabular}{|c|c|c|c|c|c|}
\hline & No & Good & Fair & Poor & $p$-value \\
\hline \multicolumn{6}{|l|}{ Penile $(n=55)$} \\
\hline OMG urethroplasty & 26 & $23(88.4 \%)$ & $1(3.8 \%)$ & $2(7.6 \%)$ & 0.20 \\
\hline Orandi procedure & 21 & $14(66.6 \%)$ & $2(9.5 \%)$ & $5(23.8 \%)$ & \\
\hline Johanson's 2 Stage & 7 & $4(57.1 \%)$ & - & $3(42.8 \%)$ & \\
\hline \multicolumn{6}{|l|}{ Bulbar $(n=212)$} \\
\hline E.P.A & 166 & $155(93.3 \%)$ & $3(1.8 \%)$ & $8(4.8 \%)$ & 0.06 \\
\hline Non-transecting & 13 & $11(84.6 \%)$ & - & $2(15.3 \%)$ & \\
\hline OMG urethroplasty & 33 & $27(81.8 \%)$ & - & $6(18.1 \%)$ & \\
\hline \multicolumn{6}{|l|}{ Pan urethral stricture $(n=57)$} \\
\hline Abdominal wall skin grafting & 31 & $23(74.1 \%)$ & $3(9.6 \%)$ & $5(16.1 \%)$ & 0.02 \\
\hline Combined tissue transfer & 14 & $11(78.5 \%)$ & - & $3(21.5 \%)$ & \\
\hline OMG urethroplasty & 12 & $9(75 \%)$ & $1(8.3 \%)$ & $2(16.6 \%)$ & \\
\hline PFUI/PFUDD $(n=222)$ & 223 & $197(88.3 \%)$ & $16(7.1 \%)$ & $10(4.4 \%)$ & NA \\
\hline
\end{tabular}

NA $=$ Not applied.

Table III: Early surgical complications.

\begin{tabular}{|c|c|c|c|c|c|c|}
\hline & Penile $(n=55)$ & Bulbar $(n=212)$ & Pan-urethral $(n=57)$ & Posterior $(n=222)$ & $\mathrm{p}$-value & $\begin{array}{l}\text { ClavianDindo } \\
\text { Classification }\end{array}$ \\
\hline Fever & $4(8.5 \%)$ & $13(7.3 \%)$ & $3(6.7 \%)$ & $15(7.7 \%)$ & 0.98 & Grade I \\
\hline Penile/ scrotal swelling & $1(1.8 \%)$ & $7(3.3 \%)$ & $5(8.8 \%)$ & $3(4 \%)$ & 0.02 & Grade I \\
\hline Wound infections & $3(6 \%)$ & $4(9 \%)$ & $2(3.5 \%)$ & $4(1.8 \%)$ & 0.36 & Grade I \\
\hline UTI & $2(3.7 \%)$ & $5(2.3 \%)$ & $2(3.5 \%)$ & $3(6.7 \%)$ & 0.62 & Grade I \\
\hline Bleeding & 0 & $3(1.4 \%)$ & $2(3.5 \%)$ & $4(1.8 \%)$ & 0.52 & Grade II \\
\hline Painful ejaculation & $1(2.5 \%)$ & $4(4.3 \%)$ & 0 & $3(2.0 \%)$ & 0.60 & Grade I \\
\hline Post-void dribbling & 0 & $3(3.2 \%)$ & $2(8.7 \%)$ & $2(1.4 \%)$ & 0.10 & Grade I \\
\hline Stress incontinence & 0 & $6(2.8 \%)$ & 0 & $15(6.8 \%)$ & 0.01 & Grade II \\
\hline Recurrence of stricture & $11(20 \%)$ & $17(8 \%)$ & $14(24.6 \%)$ & $24(10.8 \%)$ & 0.002 & Grade III B \\
\hline Chordae & $3(5.5 \%)$ & $1(0.5 \%)$ & $1(1.8 \%)$ & $4(1.7 \%)$ & 0.08 & Grade III B \\
\hline
\end{tabular}

$U T I=$ Urinary tract infection

location of stricture in the vast majority of cases, as shown in Table III. There were no deaths in this series.

Preoperatively, erectile function assessment showed poor or no erections in $155(28.3 \%)$ patients while posturethroplasty, erectile dysfunction (ED) was reported by $214(39.1 \%)$ patients at 3-6 months follow-up. This figure improved spontaneously at one year follow-up in 29 patients leaving 185 (34\%) with ED. So, the difference of weak/no erections in pre- and post-urethroplasty was $29(5.3 \%)$ patients. In these, ED persisted after urethroplasty and needed treatment.

The assessment of fertility showed that 91 (16.7\%) patients were married and fertile before urethroplasty, while post-urethroplasty, $74(13.5 \%)$ patients retained this function. The fertility status was assessed in only married patients and who had at least 1 year of follow-up.

\section{DISCUSSION}

Urethral stricture management has evolved over the last few decades. In the developed world, most of the strictures are managed in the dedicated centres by experts with special interest in reconstruction with good outcomes. The referral pathways are strictly regulated allowing management in the specialist units.
On the contrary, emerging world has generally not been able to embrace the sub-specialisation. Most patients with this condition receive a variety of interventions from novices ranging from aggressive attempts at dilatation to reconstruction. These interventions generally aggravate the consequences of injury and impact on the final outcomes when patient ultimately reaches the specialised units. ${ }^{11}$

This series is from a centre which has embraced the practice of sub-specialisation by establishing a dedicated urethral clinic. The authors reported outcomes of management of 546 patients, all males with mean age of 32 years. Peak age distribution in the previously reported cohort of 1,600 patients with urethral stricture was 41-50 years with a mean of 45 years. ${ }^{1}$ These figures suggest that increasingly younger people are involved in developing countries.

A large majority of patients in this cohort were rural dwellers, injured in farming accidents (tractors), road accidents or falls from trees or ceilings. The urethroplasty was performed in $16 \%$ of patients who attended the unit during the above period. This statistics may suggest that urethroplasty was offered to a limited number of cases and a vast majority had endoscopic management. ${ }^{12}$ However, the majority of the strictures seen in the clinic are grade I and II involving limited 
mucosal scarring which can be treated by endoscopic methods.

The etiology of the strictures varied in different segments of the urethra and in different parts of the world. A study from Mexico revealed that iatrogenic trauma due to catheterisation and urethral instrumentation was the commonest cause accounting for $73 \%$ of urethral strictures. ${ }^{12}$ In another study on etiology of strictures included, hypospadias repair in penile, idiopathic in bulbous, post-instrumentation in pan-urethral and PFUI in posterior strictures. ${ }^{13}$

In this cohort, a significant number of penile urethral strictures $(38.8 \%)$ were treated using penile skin flaps because of unsuitability of OMG due to changes caused by beetle nut chewing. Although our institutional preference has been a dorsal inlay OMG urethroplasty for penile strictures, in 7 patients we opted for the lateral or ventral onlay grafts as complete obliteration of the urethral lumen made inlay grafts impractical. We elected to perform two-stage Johanson's urethroplasty in postinfective strictures and patients with cavities and false passages and those with lichen sclerosis.

In this series, Orandi procedure gives success rate of $66 \%$, which is inferior compared to OMG urethroplasty. In a study from Netherland, De La Rosette et al. reported $32 \%$ recurrence rate and $20 \%$ fistula formation. 14 Patients with significant comorbidities and infective cavities in urethra were managed with perineal urethrostomy as the first stage of Johanson's urethroplasty in seven patients. 20 Out of these, five requested for $2^{\text {nd }}$ stage Johanson's, done six months after the first stage but two of them with age $>70$ years opted for perineal urethrostomy, which needed intermittent dilatation with Nelton catheter.

Length of strictures in the bulbar urethra ranged from $1-7 \mathrm{~cm}$ with mean of $2.43 \pm 1.26 \mathrm{~cm}$. At the study centre, 1-2 $\mathrm{cm}$ bulbur strictures were treated with EPA urethroplasty if DVIU was not successful, is non-traumatic strictures; 15 but in traumatic bulbur strictures, EPA was the primary option in 166 (78.3\%) patients. In younger patients, even strictures as long as $3-4 \mathrm{~cm}$ can be managed with this technique with excellent outcomes. ${ }^{16}$ In this series, good results were achieved in $93 \%$ of cases after EPA urethroplasty, a success rate which matches results from other reported series. ${ }^{17}$

Non-transecting urethroplasty (NTU) was undertaken in $13(6.1 \%)$ of bulbar strictures. Most suitable patients for this procedure are those with strictures $1-1.5 \mathrm{~cm}$ in length and minimal spongio-fibrosis. There are theoretical advantages of lower risk of ED because of preservation of blood supply. The results in this series were inferior to EPA urethroplasty. This may be attributable to poor selection of cases including those with traumatic strictures in whom the diseased part of urethra was not excised fully. Another series on NTU of 75 patients, with short bulbar and posterior strictures, reported $8 \%$ recurrence and $21.9 \%$ de-novo-ED which improved at 12 months follow up in all patients except one. ${ }^{18}$

OMG was used in 33 patients with bulbar strictures $>4 \mathrm{cms}$. Grafts were harvested from the inner cheek and lower lip in all except 3 , which were harvested from the dorso-lateral aspect of tongue. Of 33 patients, 8 had augmented anastomosis with dorsal onlay patch. In the remainder, stricturotomy and OMG patch was used either as a dorsal or lateral onlay in 12 and 13 patients, respectively. Good outcomes were obtained in $81.87 \%$. In one meta-analysis, OMG substitution urethroplasty reported success rate of $88.1 \%$ and no difference between dorsal and ventral onlay techniques. 18,19

Successful management of pan-urethral strictures required two-stage urethroplasty in early phase of our learning curve. 20 However, with increasing experience, we treated patients with a single-stage reconstruction. These strictures were treated either by penile skin flaps or OMG or combination of the techniques i.e. penile skin flaps for penile and OMG for bulbar as reported in another study. 21

In pan-urethral strictures, in 31 (54\%) patients, abdominal wall full-thickness skin graft was used for urethral reconstruction. It was more relevant in our set-up. Use of abdominal wall skin for repair of urethral strictures as an alternative to OMG has been reported in another study. 22

In 12 patients $(21 \%)$ with pan-urethral strictures, OMG urethroplasty was performed if the length of the stricture was upto $6 \mathrm{~cm}$. A subgroup of $14 / 57$ (24.5\%) patients underwent combined tissue transfer technique as the authors believed that repairing strictures $>6 \mathrm{~cm}$ with OMG alone increases the morbidity. Berglund et al. however, reported a series of 18 patients with a mean length of $15.1 \mathrm{~cm}$ using combined OMG and genital skin with good success. ${ }^{21}$

PFUI strictures were managed by excision and bulboprostatic anastomosis urethroplasty. Additional maneuvers used were mobilisation of urethra, separation of the crura and partial pubectomy, wherever necessary. Rerouting of urethra, however, was not required in any of the patients. Good outcomes were achieved in $88.3 \%$. In PFUl group, 10 patients $(4.4 \%)$ had poor results just after operation while other $13(6 \%)$ developed recurrence of stricture making a total of $23(10.3 \%)$ recurrence rate, they were managed by either redo urethroplasty DVIU or by Mitrofenoff continent diversion in 2 cases. Incontinence rates in this cohort of PFUI are comparable to other reported series.22,23 The overall success reported in other series of PFUI repairs ranges from $90-98 \% .22,23$ We believe that relatively inferior outcomes in our series may partly be attributed to differences in the definition of 
success and unsuccessful multiple interventions in the past at other centres. ${ }^{23}$ Morey et al. reported success rate of $93 \%$, although $11 \%$ patients amongst successful cohort required DVIU and 3\% were considered failed. ${ }^{23}$ In this study, any postoperative surgical intervention was considered as failure; whereas, in Morey et al. study, a single DVIU after urethroplasty was not considered as failure.

The adverse events were encountered in 251/546 $(45.9 \%)$ patients in this series. In another series reporting on the complications, showed early minor complications in $44 \%$ while late major complications in $22 \% .{ }^{23} \mathrm{Al}$-Qudah et al. reported $39 \%$ early and $40 \%$ late complications. ${ }^{24}$ Majority of these were minor (97\%) and resolved without intervention or late consequences. Only $3 \%$ of the early complications were major.

In the present study, erectile functions and fertility status were evaluated before and after urethroplasty. There was spontaneous improvement in erectile function at 12 months in the majority of patients but $30(10.8 \%)$ had persistence of ED after urethroplasty. This reported $10.8 \%$ ED in this series is little higher than the reported incidence of $2-5 \%$ by Blaschko et al. 12 This impact of urethroplasty on ED needs further research.

In this study, 91 patients were fertile before urethroplasty, rest were not married or did not complete followup of $>1$ year. Seventy-four remained fertile after the procedure. Effect of urethroplasty on fertility is poorly researched. Whether trauma or urethroplasty procedure causes obstruction to the ejaculatory ducts is not known and hence, needs further study.

\section{CONCLUSION}

This is the largest series reported on urethroplasty outcomes from Pakistan. The best results of reconstruction were achieved with EPA urethroplasty followed by OMG urethroplasty. Limited resources are the biggest challenge in functional rehabilitation in the developing countries.

\section{PATIENTS' CONSENT:}

Patients' consents were obtained during the procedures as part of the patient care protocol and to use the data for research purpose.

\section{CONFLICT OF INTEREST:}

Authors declared no conflict of interest.

\section{AUTHORS' CONTRIBUTION:}

$\mathrm{MH}$, MSK: Conception and designing, collection and analysis of data, primary drafting of the paper and final approval.

$\mathrm{ML}, \mathrm{AH}$ : Acquisition of data, critical review of the paper. SAAN, SAHR: Critical review and final approval of the manuscript.

\section{REFERENCES}

1. Hussain M, Askari H, Lal M, Naqvi SAA, Rizvi SAH. Experience at a stricture clinic in a developing country. $J$ Pak Med Assoc 2013; 63:234-8.

2. Latini JM, Arinch MC, Brandes SB, Change JY, Rosantein D, Chapter I. Epidemiology, etiology, anatomy and nomenclature of urethral stenosis, strictures and pelvic fracture urethral disruption Injuries. Urology 2013; 09:009.

3. Palminteri E. Stents and urethral strictures: A lesson learned? Eur Urol 2008; 54:498-500.

4. Hussain M. Urethral stricture disease: An old disease with newer treatments. J Pak Med Assoc 2008; 58:227-8.

5. Carson CC. Urethroplasty: A model for international progress in urology. Contemp Urol 2006; 18:11.

6. Erickson BA, Ghareeb GM. Definition of successful treatment and optional follow-up after urethral reconstruction for urethral stricture disease. Urol Clinic N Am 2017; 44:1-9.

7. Andrich DE, Mundy AR. Non-transecting anastomotic bulbar urethroplasty: A preliminary report. BJU Int 2012; 109:1090-4.

8. Hillary $\mathrm{CJ}$, Osman $\mathrm{NI}$, Chapple $\mathrm{CR}$. Current trends in urethral stricture management. Asian J Urol 2014; 1:46-54.

9. Al-Ali M, Al-Hajaj R. Johanson's staged urethroplasty revisited in the salvage treatment of 68 complex urethral stricture patients: presentation of total urethroplasty. Eur Urol 2001; 39: 268-71.

10. Wessells H, Morey AF, McAninch JW. Single stage reconstruction of complex anterior urethral strictures: combined tissue transfer techniques. J Urol 1997; 157:1271-4.

11. Webster GD, Peterson AC. Simple perineal and elaborated perineal posterior urethroplasty. Arab J Urol 2015; 13:17-23.

12. Blaschko SD, Sanford MT, Schlomer BJ, Alwaal A, Yang G, Villata JD, et al. The incidence of erectile dysfunction after pelvic fracture urethral injury: A Systematic review and meta analysis. Arab J Urol 2015; 13:68-74.

13. Cisneros-Chavez R, Aragon-Castro MA, Morales-Ordaz O. Urethral stricture: Etiology and treatment. Experience at the Centro Medico ISSEMYM. Rev MexUrol 2013; 73:229-36.

14. de la Rosette JJ, DeVries JDM, Lock MTWT, Debruyne FMJ. Urethroplasty using the pedicledisland flap technique in complicated urethral strictures. J Urol 1991; 146:40-2.

15. Wright $\mathrm{JL}$, Wessells $H$, Nathens $A B$, Hollingworth W. What is the most cost-effective treatment for 1 to $2-\mathrm{cm}$ bulbar urethral strictures: societal approach using decision analysis. Urology 2006; 67:889-93.

16. Terlecki RP, Steel MC, Valadez C, Moray AF. Grafts are unnecessary for proximal bulbar reconstruction. J Urol 2010; 184: 2395-9.

17. Altahawy EA, Virasoro R, Schlossberg SM, McCammon KA, Jordan $\mathrm{GH}$. Long term follow-up for excision and primary anastomosis for anterior urethral strictures. J Urol 2007; 117: $1803-6$

18. Lumen N, Poelaert F, Oosterlinck W. Nontransecting anastomotic repair in urethral reconstruction: Surgical and functional outcome. J Urol 2016; 196:1679-84.

19. Wang K, Miaox X, Wang L, Li H. Dorsal onlay vs. ventral onlay 
urethroplasty for anterior urethral strictures: A meta-analysis. Urol Int 2009; 83:342-8.

20. Mori RL, Angermeier KW. Staged urethroplasty in the management of complex anterior urethral stricture disease. Transl Androl Urol 2015; 4:29-34.

21. Berglund RK, Angermeier KW. Combined buccal mucosa graft and genital skin flap for reconstruction of extensive anterior urethral strictures. Urology 2006; 68:707-10.
22. Meeks JJ, Erickson BA, Fetchev P, Crawford SE, Fine NA, Gonzalez CM. Urethroplasty with abdominal skin grafts for long segment urethral stricture. J Urol 2010; 183:1880-4.

23. Morey AF, McAninch JW. Reconstruction of posterior urethral disruption injuries: Outcomes analysis in 82 patients. $J$ Urol 1997; 157:506-10.

24. Al-Qudah HS,Santuccai RA. Extended complications of urethroplasty. Int Braz J Urol 2005; 31:315-23.

....々.... 